\title{
Biogeochemical dynamics of iron minerals controlling transport and bioavailability in freshwater systems
}

Druschel, G.K..${ }^{1}$, SHUKLE, J. ${ }^{1}$, KUREK. M.R. ${ }^{1}$, WiLKeS, A. $^{2}$, NuZzIO, D. ${ }^{3}$, SCHroth, A. ${ }^{2}$

${ }^{1}$ Indiana University Purdue University Indianapolis; Dept. of Earth Sciences; Indianapolis, IN. USA. gdrusche@iu.edu ${ }^{2}$ University of Vermont, Dept. of Geology, Burlington, VT

${ }^{3}$ Analytical Instrument Systems, Ringoes, NJ.

Redox biogeochemistry is inherently dynamic and is often defined by sharp gradients in time and space, constrained by a network of physical, chemical, and biological reactions. We have been investigating the spatial and temporal gradients of oxygen, manganese, and iron through porewater electrochemistry and solid-state mineral characterization in several field systems and controlled mesocosm experiments. Phosphorus (P) mobility is often tied to iron oxide mineralogy and the redox biogeochemical reactions governing their formation, dissolution, ripening, and recrystallization kinetics. Trace metal mobility, and the mobility of arsenic are also tightly coupled to iron mineralization. Coupled spatio-temporal shifts in porewater redox gradients that are transient, periodic, or otherwise variable create a zone of higher dynamic activity that impacts iron mineralization and mobility of associated forms. Microbial metabolic reactions are intimately tied to these redox reactions, when the timing is appropriate that the metabolism outcompetes the abiotic reactions.

Recent advances in electrochemical devices (Analytical Instrument Systems) are making possible longer deployments of electrochemical sensors to measure oxygen, sulfur, manganese, and iron chemistry and mineralogy over seconds time scale with micron spatial resolution. The appropriate temporal scaling to 'capture' transitions, changes in frequency, and chaotic events is likely variable in different types of systems, but can be determined. Field observations from a shallow freshwater lake, mountain streams, hydrothermal systems, and lab mesocosm experiments (where external forcing of gases can manipulate timing of redox transitions) indicate fluctuations in redox porewater geochemistry are tied to mineralization. This in turn is tied to changes in mineral size and crystallinity that has a significant impact on nutrient and metal mobility. We will review approaches to observing the spatial and temporal dynamics of these key biogeochemical interactions, and field and lab observations demonstrating the links between biotic and abiotic redox cycling, mineralization, and biotic activity. 